\title{
Biomedical Engineering, Medical Engineering, and Bioengineering: Different BME Programmes in Hong Kong
}

\author{
Yong-Ping Zheng \\ Interdisciplinary Division of Biomedical Engineering, The Hong Kong Polytechnic University, Hong Kong SAR, China \\ yongping. zheng@ieee.org
}

BME undergraduate education in Hong Kong started with a $\mathrm{BSc}$ (Hons) programme in Prosthetics and Orthotics in the Jockey Club Rehabilitation Engineering in the Hong Kong Polytechnic University (PolyU) in 1995. This programme was restructured and named as Health Technology in 2000, and further re-named as Biomedical Engineering in 2005. The team offering this programme has a strong track record in rehabilitation engineering, and recently in biomedical imaging, bioinstrumentation, and bionanotechnologies. PolyU BME programme used to be in the Faulty of Health and Social Sciences. As a strategic development of the university and promote interdisciplinary research, the Interdisciplinary Division of Biomedical Engineering was established under the Faculty of Engineering as its $6^{\text {th }}$ department in 2012.

Currently there are other three universities offering BME undergraduate programmes. The University of Hong Kong (HKU) offers a BSc(Hons) programme in Medical Engineering, starting from 2002. The Chinese University of
Hong Kong (CUHK) offers a BSc (Hons) programme in Biomedical Engineering, since 2010. Both BME programmes in HKU and CUHK are offered under the collaboration between their Faculties of Engineering and Medicine. Recently, the City University of Hong Kong launched a BSc(Hons) programme in Bioengineering in 2012. PolyU, CUHK, and the Hong Kong University of Science and Technology (HKUST) offer MSc programmes in biomedical or bioengineering. The undergraduate BME programmes offered by different Hong Kong universities have slightly different focused areas, well linked with the strengths of their faculty members. With more BME graduates trained by the universities, it becomes important to understand related job market. BME graduates in Hong Kong work in variety of sectors, including further studies, hospitals, government departments, medical device industries for R\&D, sales, maintenance, project managing, hospitals, etc. The medical device industries in Hong Kong grow very fast, as it needs high-value added areas like medical device to re-develop its industry.

\footnotetext{
The original version of this chapter was inadvertently published with an incorrect chapter pagination 1324 and DOI 10.1007/978-3-31932703-7_254. The page range and the DOI has been re-assigned. The correct page range is 1330 and the DOI is 10.1007/978-3-31932703-7 255. The erratum to this chapter is available at DOI: 10.1007/978-3-319-32703-7_260
}

E. Kyriacou et al. (eds.), XIV Mediterranean Conference on Medical and Biological Engineering and Computing 2016, IFMBE Proceedings 57, 